\title{
Electrodiffusive model for neuronal and astrocytic ion concentration dynamics
}

\author{
Geir Halnes, Ivar Østby, Klas H Pettersen, Stig W Omholt, Gaute T Einevoll \\ From Twenty Second Annual Computational Neuroscience Meeting: CNS*2013 \\ Paris, France. 13-18 July 2013
}

Electrical signaling in neurons is typically modeled using the cable equation, where dendrites or axons are represented as one-dimensional electrical cables [1]. The cable model is based on the assumptions that the electrical currents along the cable are negligibly affected by (i) diffusion (due to ion concentration gradients), and (ii) variation in resistivities (due to varying ion concentrations). An electrodiffusive model, based on the Nernst-Planck equations, has been developed for situations when these assumptions do not hold [2]. Like the standard cable model, the electrodiffusive model assumes that transport phenomena are essentially one-dimensional. Unlike the standard cable model, the electrodiffusive model explicitly includes ionconcentration dynamics and its effect on diffusive currents and resistivities.

A limitation with the model [2] is that it only considered intracellular dynamics, whereas extracellular conditions were assumed to be constant. The extracellular space (ECS) comprises only about $20 \%$ of the total tissue volume, whereas the remaining $80 \%$ is the intracellular space (ICS) of various cells. When groups of cells perform similar functions simultaneously, the impact on ionic concentrations may therefore be of the same order in the ICS and ECS. For instance, during periods of intense neural signaling, the extracellular $\mathrm{K}^{+}$-concentration may locally increase by several millimolars. Clearance of excess $\mathrm{K}^{+}$ likely depends partly on diffusion in the ECS, partly on local uptake via astrocytic K+-uptake mechanisms, and partly by intracellular transport within astrocytes [3]. To model such processes, we need an electrodiffusive formalism that includes both the ICS and ECS explicitly.

Here, we derive a simple, general mathematical framework for modeling the dynamics of the membrane potential

\footnotetext{
* Correspondence: geir.halnes@umb.no

Dept. of Mathematical Sciences and Technology, Norwegian University of Life Sciences, Ås, 1432, Norway
}

$\left(v_{M}\right)$ and the ion concentrations $\left(c_{k}\right)$ for a set $(k)$ of ionic species in an intra- and extracellular domain. The formalism is based on the constraint of electroneutrality, except in the thin Debye-layers surrounding the capacitive membrane. Like the one-domain model [2], the formalism ensures (i) a consistent relationship between $v_{M}$ and $c_{k}$, and (ii) accounts for diffusion and concentration dependent variations in resistivities. Unlike the one-domain model, the formalism ensures (iii) global particle/charge conservation, and (iv) that the charges on either side of a piece of membrane must be equal in magnitude and opposite in sign. The latter constraint is implicit when the membrane is assumed to be a parallel plate capacitor, an assumption made in most models of excitable cells (see e.g., $(1-3,16)$ ).

The formalism was implemented in a model of ionic exchange between astrocytes and the extracellular space. By simulations, we estimated the contribution of astrocytes in $\mathrm{K}+$ removal from high concentration regions, and revealed a (to our knowledge) novel mechanism that astrocytes may utilize to remove $\mathrm{K}^{+}$from extracellular high concentration regions.

\section{Acknowledgements}

The project was supported from the eScience program (eNEURO) of the Research Council of Norway.

Published: 8 July 2013

\section{References}

1. Rall W: Core conductor theory and cable properties of neurons. Handbook of Physiology: The nervous system , E. R. Kandel 1977, 39-97, American Physiological Society, Bethesda, Md.

2. Qian N, Sejnowski TJ: An electro-diffusion model for computing membrane potentials and ionic concentrations in branching dendrites, spines and axons. Biological Cybernetics 1989, 62:1-15.

3. Chen KC, Nicholson C: Spatial buffering of potassium ions in brain extracellular space. Biophysical journal 2000, 78(6):2776-97.

doi:10.1186/1471-2202-14-S1-P122

Cite this article as: Halnes et al:: Electrodiffusive model for neuronal and astrocytic ion concentration dynamics. BMC Neuroscience 2013

14(Suppl 1):P122.

\section{() Biomed Central}

